\title{
The average representation - a cornucopia of power indices?
}

\author{
Serguei Kaniovski* and Sascha Kurz ${ }^{\dagger}$
}

November 12, 2018

\begin{abstract}
For the classical power indices there is a disproportion between power and relative weights, in general. We introduce two new indices, based on weighted representations, which are proportional to suitable relative weights and which also share several important properties of the classical power indices. Imposing further restrictions on the set of representations may lead to a whole family of such indices.
\end{abstract}

Keywords: voting power, power indices, proportionality, weighted majority games, representations

JEL: C71, D71

\section{Introduction}

The observation, that the distribution of power in weighted majority games differs from the distribution of voting weights, has motivated the development of a theory of power measurement. A famous example considers three voters, having 50, 49, and 1 votes. The motion is passed if the total number of votes in favor exceeds 50. Since any two voters, but none alone, can pass the motion, any reasonable power index assigns equal power to all three voters.

The power distribution in the above example markedly differs from the relative weight distribution: $\left\|\left(\frac{50}{100}, \frac{49}{100}, \frac{1}{100}\right)-\left(\frac{1}{3}, \frac{1}{3}, \frac{1}{3}\right)\right\|_{1}=\frac{97}{150} \approx 0.65$. One reason for this disagreement is the fact that voting weights are not unique. For three voters having 100 votes in total, there are 1176 integer valued weight distributions being extendable by a suitable quota to the same game. An example is given by the weights 34, 33, 33 and a quota of 60 . There are 13872 possibilities to represent the game if the (integer) quota is considered to be part of the specification.

Having plenty of representations to choose from, can we choose voting weights that accurately reflect power measured by some index? The theoretical literature shows that, in general, we cannot, although there are particular cases when

\footnotetext{
*Austrian Institute of Economic Research (WIFO), Austria. E-mail: serguei.kaniovski@wifo.ac.at.

${ }^{\dagger}$ Department of Mathematics, University of Bayreuth, Germany. E-mail: sascha.kurz@uni-bayreuth.de.(Corresponding author.)
} 
it may be possible. Recently Houy \& Zwicker, 2014 have characterized a class of weighted majority games, which admit a representation using their respective Banzhaf distribution. For the nucleolus, we known that $\left(q\left(x^{\star}, v\right), x^{\star}\right)$ is a representation of a constant-sum weighted majority game, where $x^{\star}(v)$ denotes the nucleolus of $v$, and $q\left(x^{\star}, v\right)$ denotes the corresponding maximum excess [Maschler et al., 2013, Theorem 20.52]. If the weights are close to the average weight of the voters, then the nucleolus is close to the relative weight distribution; the two may even coincide under certain conditions, see Kurz et al., 2014. The existing power indices are not representation compatible. One exception is the recently introduced minimum sum representation (MSR) index Freixas \& Kaniovski, 2014. Also the Colomer index Colomer \& Martinez, 1995 uses weights of a majority game in its specification, but the index depends on the given representation, instead of the underlying simple game.

In this paper, we show how to construct representation compatible power indices for weighted majority games.

\section{Games and representations}

A simple game $v$ is a mapping $v: 2^{N} \rightarrow\{0,1\}$, where $N=\{1, \ldots, n\}$ is the set of voters, such that $v(\emptyset)=0, v(N)=1$, and $v(S) \leq v(T)$ for all $S \subseteq T \subseteq N$. The subsets $S \subseteq N$ are called coalitions of $v$. We call a coalition $S$ winning if $v(S)=1$, and losing otherwise. If $S$ is a winning coalition and none of its proper subsets is winning, it is called a minimal winning coalition. Similarly, if $T$ is a losing coalition and none of its proper supersets is losing, it is called a maximal losing coalition. A voter $i \in N$ with $v(S)=v(S \cup\{i\})$ for all $S \subseteq N \backslash\{i\}$ is called a dummy (or null player by some authors).

A weighted majority game is a simple game $v$, such that there exist real numbers $w_{1}, \ldots, w_{n} \geq 0$ and $q>0$ with $\sum_{s \in S} w_{s} \geq q$ for all winning coalitions $S \subseteq N$ and $\sum_{s \in T} w_{s}<q$ for all losing coalitions $T \subseteq N$. We write $v=\left[q ; w_{1}, \ldots, w_{n}\right]$, where we call $\left(q, w_{1}, \ldots, w_{n}\right)$ a representation of $v$. A weight vector $\left(w_{1}, \ldots, w_{n}\right)$ is called feasible for $v$, if there exists a quota $q$ such that $\left(q ; w_{1}, \ldots, w_{n}\right)$ is a representation of $v$. For our initial example $[2 ; 1,1,1]$, the weight vector $(49,48,3)$ is feasible, while $(50,25,25)$ is not feasible.

We collect some basic well known facts about representations of weighted majority games:

Lemma 1 Each weighted majority game $v$ admits a representation $\left(q, w_{1}, \ldots, w_{n}\right)$ with $w_{1}, \ldots, w_{n} \geq 0, q>0$, and

(1) $\sum_{i=1}^{n} w_{i}=1, q \in(0,1]$;

(2) $\sum_{i=1}^{n} w_{i}=1, q \in(0,1]$, and $w_{i}=0$ for all dummies;

(3) $q \in \mathbb{N}, w_{i} \in \mathbb{N}$.

We call a representation satisfying the conditions of (1) a normalized representation, and those satisfying the conditions of (3) an integer representation. A 
representation with $w_{i}=0$ for all dummies $i \in N$ is called dummy revealing 1

Algorithmic checks and descriptions whether a given simple game is weighted have been studied extensively in the literature, see e.g. [Taylor \& Zwicker, 1999].

Lemma 2 The set of all normalized weight vectors $w \in \mathbb{R}_{\geq 0}^{n}, \sum_{i=1}^{n} w_{i}=1$ being feasible for a given weighted majority game $v$ is given by the intersection $\sum_{i \in S} w_{i}>\sum_{i \in T} w_{i}$ for all pairs $(S, T)$, where $S$ is a minimal winning and $T$ is a maximal losing coalition of $v$.

Lemma 3 The set of all normalized representations $(q, w) \in \mathbb{R}_{\geq 0}^{n+1}, q \in(0,1]$, $\sum_{i=1}^{n} w_{i}=1$ representing a given weighted majority game $v$ is given by the intersection $\sum_{i \in S} w_{i} \geq q, \sum_{i \in T} w_{i}<q$ for all minimal winning coalitions $S$ and all maximal losing coalitions $T$.

\section{Power indices}

Let $\mathcal{S}_{n}$ denote the set of simple games on $n$ voters, and $\mathcal{W}_{n}$ the set of weighted majority games on $n$ voters. A power index for $\mathcal{C} \in\left\{\mathcal{S}_{n}, \mathcal{W}_{n} \mid n \in \mathbb{N}\right\}$ is a mapping $g: \mathcal{C} \rightarrow \mathbb{R}^{n}$, where $n$ denotes the number of voters in each game of $\mathcal{C}$. Usually, we define a vector-valued power index by defining its elements $g_{i}$, the power of a voter $i \in N$. The Shapley-Shubik index is given by

$$
S S I_{i}(v)=\sum_{S \subseteq N \backslash\{i\}} \frac{|S| !(|N|-1-|S|) !}{|N| !} \cdot(v(S \cup\{i\})-v(S)) .
$$

Definition 4 Let $g: \mathcal{C} \rightarrow \mathbb{R}^{|N|}=\left(g_{i}\right)_{i \in N}$ be a power index for $\mathcal{C}$. We say that

(1) $g$ is symmetric: if for all $v \in \mathcal{C}$ and any bijection $\tau: N \rightarrow \tau$ we have $g_{\tau(i)}(\tau v)=g_{i}(v)$, where $\tau v(S)=v(\tau(S))$ for all $S \subseteq N$;

(2) $g$ is positive: if for all $v \in \mathcal{C}$ we have $g_{i}(v) \geq 0$ and $g(v) \neq 0$;

(3) $g$ is efficient: if for all $v \in \mathcal{C}$ we have $\sum_{i=1}^{n} g_{i}(v)=1$;

(4) $g$ satisfies the dummy property: if for all $v \in \mathcal{C}$ and all dummies $i$ of $v$ we have $g_{i}(v)=0$.

The Shapley-Shubik index is symmetric, positive, efficient, and satisfies the dummy property.

In this paper, we consider power indices that can be defined on the set of weighted games. Having proportionality of weights and power in mind we define:

Definition 5 A power index $g: \mathcal{W}_{n} \rightarrow \mathbb{R}^{n}$ for weighted majority games on $n$ voters is called representation compatible if $\left(g_{1}(v), \ldots, g_{n}(v)\right)$ is feasible for all $v \in \mathcal{W}_{n}$

\footnotetext{
${ }^{1}$ The problem of checking whether a voter is a dummy in a general (integer) representation it coNP-complete Chalkiadakis et al., 2011, Theorem 4.4].
} 
We remark that the Shapley-Shubik index is representation compatible for $\mathcal{W}_{n}$ if and only if $n \leq 3$. Below, we list the weighted majority games with up to 3 voters (in minimum sum integer representation), and the representation given by the Shapley-Shubik vector.

$$
\begin{aligned}
& {[1 ; 1]=[1 ; 1] \quad[1 ; 1,0,0]=\left[\frac{6}{6} ; \frac{6}{6}, \frac{0}{6}, \frac{0}{6}\right] \quad[2 ; 1,1,1]=\left[\frac{4}{6} ; \frac{2}{6}, \frac{2}{6}, \frac{2}{6}\right]} \\
& {[1 ; 1,0]=\left[\frac{2}{2} ; \frac{2}{2} ; \frac{0}{2}\right] \quad[1 ; 1,1,0]=\left[\frac{3}{6} ; \frac{3}{6}, \frac{3}{6}, \frac{0}{6}\right] \quad[3 ; 1,1,1]=\left[\frac{6}{6} ; \frac{2}{6}, \frac{2}{6}, \frac{2}{6}\right]} \\
& {[1 ; 1,1]=\left[\frac{1}{2} ; \frac{1}{2}, \frac{1}{2}\right] \quad[2,1,1,0]=\left[\frac{6}{6} ; \frac{3}{6}, \frac{3}{6}, \frac{0}{6}\right] \quad[3 ; 2,1,1]=\left[\frac{5}{6} ; \frac{4}{6}, \frac{1}{6}, \frac{1}{6}\right]} \\
& {[2,1,1]=\left[\frac{2}{2} ; \frac{1}{2}, \frac{1}{2}\right][1 ; 1,1,1]=\left[\frac{2}{6} ; \frac{2}{6}, \frac{2}{6}, \frac{2}{6}\right] \quad[2 ; 2,1,1]=\left[\frac{2}{6} ; \frac{4}{6}, \frac{1}{6}, \frac{1}{6}\right]}
\end{aligned}
$$

For $n \geq 4$, consider the example $v=[3 ; 2,1,1,1,0, \ldots, 0]$ with $n-4$ dummies. The Shapley-Shubik index of $v$ is given by $\left(\frac{1}{2}, \frac{1}{6}, \frac{1}{6}, \frac{1}{6}, 0, \ldots, 0\right)$. Since $\{2,3,4\}$ is a winning coalition with weight $\frac{1}{2}$, and $\{1\}$ is a losing coalition with an equal weight, the Shapley-Shubik vector cannot be feasible.

\section{Representation compatible power indices}

The aim of this paper was to design power indices, which are representation compatible. Given a set of representations of the same weighted majority game $v$, each convex combination gives a representation of $v$ too. So a simple idea to construct a representation compatible power index is to specify the set of representations and the weights of the convex combination.

\subsection{The average weight index}

Definition 6 The average weight index of a weighted majority game $v$ is the average of all normalized ${ }^{2}$ weight vectors which are feasible for $v$.

For $[3 ; 2,1,1]$ we have already mentioned the sets of minimal winning and maximal losing coalitions. Applying Lemma 2 gives the constraints

$$
\begin{aligned}
w_{1}+w_{2}>w_{1} & \Longleftrightarrow w_{2}>0 \\
w_{1}+w_{3}>w_{1} & \Longleftrightarrow w_{3}>0 \\
w_{1}+w_{2}>w_{2}+w_{3} & \Longleftrightarrow w_{1}>w_{3} \\
w_{1}+w_{3}>w_{2}+w_{3} & \Longleftrightarrow w_{1}>w_{2},
\end{aligned}
$$

\footnotetext{
${ }^{2}$ Taking all weight vectors instead of the normalized ones does not make a difference.
} 
in addition to $w_{1}, w_{2}, w_{3} \geq 0$ and $w_{1}+w_{2}+w_{3}=1$. Eliminating the variable $w_{3}$ via $w_{3}=1-w_{1}-w_{2}$ and removing the redundant constraints leaves

$$
\begin{aligned}
w_{2}>0 & \Longleftrightarrow w_{2}>0 \\
1-w_{1}-w_{2}>0 & \Longleftrightarrow w_{2}<1-w_{1} \\
w_{1}>1-w_{1}-w_{2} & \Longleftrightarrow w_{2}>1-2 w_{1} \\
w_{1}>w_{2} & \Longleftrightarrow w_{2}<w_{1}
\end{aligned}
$$

Since we need $1-2 w_{1}<w_{1}$ and $1-w_{1}>0$, we have $w_{1} \in\left(\frac{1}{3}, 1\right)$. For $w_{1} \in\left(\frac{1}{3}, \frac{1}{2}\right)$ the conditions condense to $w_{2} \in\left(1-2 w_{1}, w_{1}\right)$ and for $w_{1} \in\left[\frac{1}{2}, 1\right)$ the conditions condense to $w_{2} \in\left(0,1-w_{1}\right)$.

The (scaled) average weight for voter 1 is given by

$$
\int_{\frac{1}{3}}^{\frac{1}{2}} \int_{1-2 w_{1}}^{w_{1}} w_{1} \mathrm{~d} w_{2} \mathrm{~d} w_{1}+\int_{\frac{1}{2}}^{1} \int_{0}^{1-w_{1}} w_{1} \mathrm{~d} w_{2} \mathrm{~d} w_{1}=\frac{1}{54}+\frac{1}{12}=\frac{11}{108} .
$$

For voter 2 we similarly obtain

$$
\int_{\frac{1}{3}}^{\frac{1}{2}} \int_{1-2 w_{1}}^{w_{1}} w_{2} \mathrm{~d} w_{2} \mathrm{~d} w_{1}+\int_{\frac{1}{2}}^{1} \int_{0}^{1-w_{1}} w_{2} \mathrm{~d} w_{2} \mathrm{~d} w_{1}=\frac{1}{48}+\frac{5}{432}=\frac{7}{216} .
$$

Since the volume of the feasible region is given by

$$
\int_{\frac{1}{3}}^{\frac{1}{2}} \int_{1-2 w_{1}}^{w_{1}} 1 \mathrm{~d} w_{2} \mathrm{~d} w_{1}+\int_{\frac{1}{2}}^{1} \int_{0}^{1-w_{1}} 1 \mathrm{~d} w_{2} \mathrm{~d} w_{1}=\frac{1}{8}+\frac{1}{24}=\frac{1}{6},
$$

we have

$$
\int_{\frac{1}{3}}^{\frac{1}{2}} \int_{1-2 w_{1}}^{w_{1}} w_{3} \mathrm{~d} w_{2} \mathrm{~d} w_{1}+\int_{\frac{1}{2}}^{1} \int_{0}^{1-w_{1}} w_{3} \mathrm{~d} w_{2} \mathrm{~d} w_{1}=\frac{1}{6}-\frac{11}{108}-\frac{7}{216}=\frac{7}{216} .
$$

Normalizing, or dividing by the volume of the feasible region, yields the power distribution $\left(\frac{11}{18}, \frac{7}{36}, \frac{7}{36}\right)$, with a norm-1-distance of $\frac{1}{9}$ to the respective ShapleyShubik vector $\left(\frac{2}{3}, \frac{1}{6}, \frac{1}{6}\right)$.

Actually we have dealt with the polyhedron $P=\left\{\left(w_{1}, w_{2}\right) \in \mathbb{R}^{2} \mid w_{2} \geq\right.$ $\left.0, w_{2} \leq 1-w_{1}, w_{2} \geq 1-2 w_{1}, w_{2} \leq w_{1}\right\}$, i.e., we have replaced strict inequalities by the corresponding non-strict inequalities, and considered the integrals $\int_{p} w_{1} \mathrm{~d} w_{1}, \int_{p} w_{2} \mathrm{~d} w$, and $\int_{p} 1 \mathrm{~d} w$. This modification is permitted since in general the polyhedron $P$ (after the elimination of variable $w_{n}$ ) is full dimensional, i.e., it has dimension $n-1$, so that the subspaces where equality hold in of of the inequalities have volume zero.

Lemma 7 For each weighted majority game $v$ there exist positive real numbers $\tilde{q}, \tilde{w}_{1}, \ldots, \tilde{w}_{n-1}$ and a parameter $\alpha>0$ such that

$$
\left(\tilde{q}+\delta_{0}, \tilde{w}_{1}+\delta_{1}, \ldots, \tilde{w}_{n-1}+\delta_{n-1}, 1-\sum_{i=1}^{n-1}\left(\tilde{w}_{i}+\delta_{i}\right)\right)
$$

is a normalized representation of $v$ for all $\delta_{i} \in[-\alpha, \alpha], 0 \leq i \leq n-1$. 
Proof. Let $\left(q, w_{1}, \ldots, w_{n}\right)$ be an integer representation of $v$, i.e. the weight of each winning coalition is at least $q$, and the weight of each losing coalition is at most $q-1$. Since $\left((n+1) q,(n+1) w_{1}+1, \ldots,(n+1) w_{n}\right)$ is also an integer representation of $v$, we additionally assume w.l.o.g. that $w_{i} \geq 1$ for all $1 \leq$ $i \leq n$. One can easily check that also $\left(q-\frac{2}{5}+\tilde{\delta}_{0}, w_{1}+\tilde{\delta}_{1}, \ldots, w_{n}+\tilde{\delta}_{n}\right)$ is a representation of $v$ for all $\tilde{\delta}_{i} \in\left[-\frac{1}{5 n}, \frac{1}{5} n\right], 0 \leq i \leq n$. With $s=\sum_{i=1}^{n} w_{i}$ let $\tilde{q}=\frac{1}{s} \cdot\left(q-\frac{2}{5}\right)$ and $\tilde{w}_{i}=\frac{1}{s} \cdot w_{i}$ for all $1 \leq i \leq n-1$. The choice of a suitable $\alpha$ is a bit technical ( $\alpha=\frac{1}{10 n s}$ does work), but its existence is guaranteed from our construction.

Definition 8 For each weighted majority game $v$ the (normalized) weight polyhedron $P^{\text {weight }}(v)$ is given by $P^{\text {weight }}(v)=\left\{w \in \mathbb{R}_{\geq 0}^{n} \mid\|w\|_{1}=1, w(S) \geq\right.$ $w(T) \forall$ min. winning $S$ and all max. losing $T\}$.

By fixing the quota at a suitable value we can directly conclude from Lemma 7 ,

Corollary 9 The $n$-1-dimensional volume of $P^{\text {weight }}(v)$ is non-zero for each weighted majority game $v$.

Lemma 10 The average weight index of a weighted majority game $v$ is given by

$$
\frac{1}{\int_{P^{\text {weight }}(v)} \mathrm{d} w} \cdot\left(\int_{P^{\text {weight }}(v)} w_{1} \mathrm{~d} w, \ldots, \int_{P^{\text {weight }}(v)} w_{n} \mathrm{~d} w\right) .
$$

We remark that the average weight representations is the center of mass of the polyhedron $P^{\text {weight }}(v)$.

\subsection{The average representation index}

As mentioned already in the introduction, one may consider the quota as being part of the weighted representation. To this end we introduce:

Definition 11 The average representation index of a weighted majority game $v$ is the average of all normalized ${ }^{3}$ representations of $v$.

Definition 12 For each weighted majority game $v$ the (normalized) representation polyhedron $P^{r e p}(v)$ is given by $P^{r e p}(v)=\left\{(q, w) \in \mathbb{R}_{\geq 0}^{n+1} \mid \sum_{i=1}^{n} w_{i}=1, q \leq\right.$ $1, w(S) \geq q \forall$ min. winning coalitions $S, w(T) \leq q \forall$ max. losing coalitions $T\}$.

Using Lemma 3 and Lemma 7 we conclude:

Lemma 13 The average representation index of a weighted majority game $v$ is given by

$$
\frac{1}{\int_{P^{r e p}(v)} \mathrm{d}(q, w)} \cdot\left(\int_{P^{r e p}(v)} w_{1} \mathrm{~d}(q, w), \ldots, \int_{P^{r e p}(v)} w_{n} \mathrm{~d}(q, w)\right) .
$$

\footnotetext{
${ }^{3}$ Taking all representations instead of the normalized ones does not make a difference.
} 
For our example $v=[3 ; 2,1,1]$ we have $P^{\mathrm{rep}}(v)=\left\{(q, w) \in \mathbb{R}_{\geq 0}^{4} \mid \sum_{i=1}^{3} w_{i}=1, w_{1}+w_{2} \geq q, w_{1}+w_{3} \geq q, w_{1} \leq q, w_{2}+w_{3} \leq q\right\}$,

and

$$
\begin{aligned}
& \int_{P_{\operatorname{rep}}(v)} \mathrm{d}(q, w)=\int_{\frac{1}{2}}^{\frac{2}{3}} \int_{1-q}^{q} \int_{q-w_{1}}^{1-q} \mathrm{~d} w_{2} \mathrm{~d} w_{1} \mathrm{~d} q+\int_{\frac{2}{3}}^{1} \int_{2 q-1}^{q} \int_{q-w_{1}}^{1-q} \mathrm{~d} w_{2} \mathrm{~d} w_{1} \mathrm{~d} q \\
&=\frac{5}{648}+\frac{1}{162}=\frac{1}{72}, \\
& \int_{P^{\operatorname{rep}(v)} w_{1} \mathrm{~d}(q, w)}=\int_{\frac{1}{2} \int_{1-q}^{\frac{2}{3}} \int_{q-w_{1}}^{q} w_{1} \mathrm{~d} w_{2} \mathrm{~d} w_{1} \mathrm{~d} q+\int_{\frac{2}{3}}^{1-q} \int_{2 q-1}^{q} \int_{q-w_{1}}^{1-q} w_{1} \mathrm{~d} w_{2} \mathrm{~d} w_{1} \mathrm{~d} q} \\
&=\frac{31}{7776}+\frac{1}{243}=\frac{7}{864}, \\
& \int_{P_{\operatorname{rep}(v)}} w_{2} \mathrm{~d}(q, w)=\int_{\frac{1}{3}}^{q} \int_{1-q}^{1-q} w_{2} \mathrm{~d} w_{2} \mathrm{~d} w_{1} \mathrm{~d} q+\int_{\frac{2}{3}}^{1} \int_{2 q-1}^{q} \int_{q-w_{1}}^{1-q} w_{2} \mathrm{~d} w_{2} \mathrm{~d} w_{1} \mathrm{~d} q \\
&=\frac{29}{15552}+\frac{1}{972}=\frac{5}{1728},
\end{aligned}
$$

so that the average representation index is given by $\left(\frac{7}{12}, \frac{5}{24}, \frac{5}{24}\right)$.

\subsection{Properties of the new indices}

The two newly introduced indices share several of the properties commonly required for a power index. Three of four properties in Definition 4 are satisfied.

Lemma 14 The average weight and the average representation index are symmetric, positive, and efficient, satisfies strong monotonicity, but do not satisfy the dummy property.

Proof. Symmetry, positivity, and efficiency are inherent in the definition of both indices. The violation of the dummy property can e.g. be seen at example $[1 ; 1,0]$.

The later shortcoming can be repaired using a quite general approach.

Lemma 15 Given a sequence of power indices $g^{n}: \mathcal{C}_{n} \rightarrow \mathbb{R}^{n}$ for all $n \in \mathbb{N}$, let $\tilde{g}^{n}: \mathcal{C}_{n} \rightarrow \mathbb{R}^{n}$ be defined via $\tilde{g}_{i}^{n}(v)=g_{i}^{m}\left(v^{\prime}\right)$ for all non-dummies $i$ and by $\tilde{g}_{j}^{n}(v)=0$ for all dummies $j$, where $m$ is the number of non-dummies in $v$ and $v^{\prime}$ arises from $v$ by dropping the dummie $A$ All $\tilde{g}^{n}$ satisfy the dummy property.

\footnotetext{
${ }^{4}$ Given a weighted majority game $v: 2^{N} \rightarrow\{0,1\}$ with $S=\{i \in N \mid i$ is dummy $\}$, we define the dummy reduced game $v^{\prime}: 2^{N \backslash S} \rightarrow\{0,1\}$ via $v^{\prime}(T)=v(T)$ for all $T \subseteq N \backslash S$.
} 
We call $\tilde{g}^{n}$ the dummy revealing version of a given sequence of power indices $g^{n}$. For the computation of the dummy revealing version we just have to compute the dummy reduced game $v^{\prime}$ and its corresponding power distribution.

\subsection{Algorithmic computation of the new indices}

The computations from Lemma 10 and Lemma 13 can easily be performed using the software package LattE [Baldoni et al., 2014], i.e., there is no need to perform the nasty case differentiations and evaluations of multi integrals, as done for our example, by hand.

\begin{tabular}{|c|c|c|c|c|c|}
\hline game & av. weight & av. rep. & game & av. weight & av. rep. \\
\hline$[1 ; 1]$ & (1) & (1) & {$[3 ; 2,1,1,0]$} & $\left(\frac{67}{120}, \frac{47}{240}, \frac{47}{240}, \frac{1}{20}\right)$ & $\left(\frac{41}{75}, \frac{31}{150}, \frac{31}{150}, \frac{1}{25}\right)$ \\
\hline$[1 ; 1,0]$ & $\left(\frac{3}{4}, \frac{1}{4}\right)$ & $\left(\frac{5}{6}, \frac{1}{6}\right)$ & {$[1 ; 1,1,1,1]$} & $\left(\frac{1}{4}, \frac{1}{4}, \frac{1}{4}, \frac{1}{4}\right)$ & $\left(\frac{1}{4}, \frac{1}{4}, \frac{1}{4}, \frac{1}{4}\right)$ \\
\hline$[1 ; 1,1]$ & $\left(\frac{1}{2}, \frac{1}{2}\right)$ & $\left(\frac{1}{2}, \frac{1}{2}\right)$ & {$[2 ; 1,1,1,1]$} & $\left(\frac{1}{4}, \frac{1}{4}, \frac{1}{4}, \frac{1}{4}\right)$ & $\left(\frac{1}{4}, \frac{1}{4}, \frac{1}{4}, \frac{1}{4}\right)$ \\
\hline$[2 ; 1,1]$ & $\left(\frac{1}{2}, \frac{1}{2}\right)$ & $\left(\frac{1}{2}, \frac{1}{2}\right)$ & {$[3 ; 1,1,1,1]$} & $\left(\frac{1}{4}, \frac{1}{4}, \frac{1}{4}, \frac{1}{4}\right)$ & $\left(\frac{1}{4}, \frac{1}{4}, \frac{1}{4}, \frac{1}{4}\right)$ \\
\hline$[1 ; 1,0,0]$ & $\left(\frac{2}{3}, \frac{1}{6}, \frac{1}{6}\right)$ & $\left(\frac{3}{4}, \frac{1}{8}, \frac{1}{8}\right)$ & {$[4 ; 1,1,1,1]$} & $\left(\frac{1}{4}, \frac{1}{4}, \frac{1}{4}, \frac{1}{4}\right)$ & $\left(\frac{1}{4}, \frac{1}{4}, \frac{1}{4}, \frac{1}{4}\right)$ \\
\hline$[1 ; 1,1,0]$ & $\left(\frac{4}{9}, \frac{4}{9}, \frac{1}{9}\right)$ & $\left(\frac{11}{24}, \frac{11}{24}, \frac{1}{12}\right)$ & {$[4 ; 2,1,1,1]$} & $\left(\frac{23}{48}, \frac{25}{144}, \frac{25}{144}, \frac{25}{144}\right)$ & $\left(\frac{139}{300}, \frac{161}{900}, \frac{161}{900}, \frac{161}{900}\right)$ \\
\hline$[2 ; 1,1,0]$ & $\left(\frac{4}{9}, \frac{4}{9}, \frac{1}{9}\right)$ & $\left(\frac{11}{24}, \frac{11}{24}, \frac{1}{12}\right)$ & {$[3 ; 2,1,1,1]$} & $\left(\frac{7}{16}, \frac{3}{16}, \frac{3}{16}, \frac{3}{16}\right)$ & $\left(\frac{43}{100}, \frac{19}{100}, \frac{19}{100}, \frac{19}{100}\right)$ \\
\hline$[1 ; 1,1,1]$ & $\left(\frac{1}{3}, \frac{1}{3}, \frac{1}{3}\right)$ & $\left(\frac{1}{3}, \frac{1}{3}, \frac{1}{3}\right)$ & {$[2 ; 2,1,1,1]$} & $\left(\frac{23}{48}, \frac{25}{144}, \frac{25}{144}, \frac{25}{144}\right)$ & $\left(\frac{139}{300}, \frac{161}{900}, \frac{161}{900}, \frac{161}{900}\right)$ \\
\hline$[2 ; 1,1,1]$ & $\left(\frac{1}{3}, \frac{1}{3}, \frac{1}{3}\right)$ & $\left(\frac{1}{3}, \frac{1}{3}, \frac{1}{3}\right)$ & {$[3 ; 2,2,1,1]$} & $\left(\frac{83}{240}, \frac{83}{240}, \frac{37}{240}, \frac{37}{240}\right)$ & $\left(\frac{103}{300}, \frac{103}{300}, \frac{47}{300}, \frac{47}{300}\right)$ \\
\hline$[3 ; 1,1,1]$ & $\left(\frac{1}{3}, \frac{1}{3}, \frac{1}{3}\right)$ & $\left(\frac{1}{3}, \frac{1}{3}, \frac{1}{3}\right)$ & {$[4 ; 2,2,1,1]$} & $\left(\frac{83}{240}, \frac{83}{240}, \frac{37}{240}, \frac{37}{240}\right)$ & $\left(\frac{103}{300}, \frac{103}{300}, \frac{47}{300}, \frac{47}{300}\right)$ \\
\hline$[2 ; 2,1,1]$ & $\left(\frac{11}{18}, \frac{7}{36}, \frac{7}{36}\right)$ & $\left(\frac{7}{12}, \frac{5}{24}, \frac{5}{24}\right)$ & {$[5 ; 2,2,1,1]$} & $\left(\frac{19}{48}, \frac{19}{48}, \frac{5}{48}, \frac{5}{48}\right)$ & $\left(\frac{23}{60}, \frac{23}{60}, \frac{7}{60}, \frac{7}{60}\right)$ \\
\hline$[3 ; 2,1,1]$ & $\left(\frac{11}{18}, \frac{7}{36}, \frac{7}{36}\right)$ & $\left(\frac{7}{12}, \frac{5}{24}, \frac{5}{24}\right)$ & {$[2 ; 2,2,1,1]$} & $\left(\frac{19}{48}, \frac{19}{48}, \frac{5}{48}, \frac{5}{48}\right)$ & $\left(\frac{23}{60}, \frac{23}{60}, \frac{7}{60}, \frac{7}{60}\right)$ \\
\hline$[1 ; 1,0,0,0]$ & $\left(\frac{5}{8}, \frac{1}{8}, \frac{1}{8}, \frac{1}{8}\right)$ & $\left(\frac{7}{10}, \frac{1}{10}, \frac{1}{10}, \frac{1}{10}\right)$ & {$[4 ; 3,1,1,1]$} & $\left(\frac{3}{5}, \frac{2}{15}, \frac{2}{15}, \frac{2}{15}\right)$ & $\left(\frac{29}{50}, \frac{7}{50}, \frac{7}{50}, \frac{7}{50}\right)$ \\
\hline$[1 ; 1,1,0,0]$ & $\left(\frac{5}{12}, \frac{5}{12}, \frac{1}{12}, \frac{1}{12}\right)$ & $\left(\frac{13}{30}, \frac{13}{30}, \frac{1}{15}, \frac{1}{15}\right)$ & {$[3 ; 3,1,1,1]$} & $\left(\frac{3}{5}, \frac{2}{15}, \frac{2}{15}, \frac{2}{15}\right)$ & $\left(\frac{29}{50}, \frac{7}{50}, \frac{7}{50}, \frac{7}{50}\right)$ \\
\hline$[2 ; 1,1,0,0]$ & $\left(\frac{5}{12}, \frac{5}{12}, \frac{1}{12}, \frac{1}{12}\right)$ & $\left(\frac{13}{30}, \frac{13}{30}, \frac{1}{15}, \frac{1}{15}\right)$ & {$[3 ; 3,2,1,1]$} & $\left(\frac{449}{840}, \frac{227}{80}, \frac{41}{420}, \frac{41}{420}\right)$ & $\left(\frac{77}{150}, \frac{41}{150}, \frac{8}{75}, \frac{8}{75}\right)$ \\
\hline$[1 ; 1,1,1,0]$ & $\left(\frac{5}{16}, \frac{5}{16}, \frac{5}{16}, \frac{1}{16}\right)$ & $\left(\frac{19}{60}, \frac{19}{60}, \frac{19}{60}, \frac{1}{20}\right)$ & {$[5 ; 3,2,1,1]$} & $\left(\frac{449}{840}, \frac{227}{840}, \frac{41}{420}, \frac{41}{420}\right)$ & $\left(\frac{77}{150}, \frac{41}{150}, \frac{8}{75}, \frac{8}{75}\right)$ \\
\hline$[2 ; 1,1,1,0]$ & $\left(\frac{5}{16}, \frac{5}{16}, \frac{5}{16}, \frac{1}{16}\right)$ & $\left(\frac{19}{60}, \frac{19}{60}, \frac{19}{60}, \frac{1}{20}\right)$ & {$[4 ; 3,2,2,1]$} & $\left(\frac{193}{480}, \frac{31}{120}, \frac{31}{120}, \frac{13}{160}\right)$ & $\left(\frac{119}{300}, \frac{77}{300}, \frac{77}{300}, \frac{9}{100}\right)$ \\
\hline$[3 ; 1,1,1,0]$ & $\left(\frac{5}{16}, \frac{5}{16}, \frac{5}{16}, \frac{1}{16}\right)$ & $\left(\frac{19}{60}, \frac{19}{60}, \frac{19}{60}, \frac{1}{20}\right)$ & {$[5 ; 3,2,2,1]$} & $\left(\frac{193}{480}, \frac{31}{120}, \frac{31}{120}, \frac{13}{160}\right)$ & $\left(\frac{119}{300}, \frac{77}{300}, \frac{77}{300}, \frac{9}{100}\right)$ \\
\hline$[2 ; 2,1,1,0]$ & & $\frac{31}{150}, \frac{31}{150}, \frac{1}{25}$ & & & \\
\hline
\end{tabular}

Table 1: The average weight and average representation index for small weighted majority games.

In Table 1 we list the average weight and the average representation index for all weighted majority games with up to 4 voters. We observe that the so-called dual games obtain the same average weight and average representation index, which can indeed be proved easily.

\section{Conclusion and future research}

We have shown how to construct power indices that respect proportionality between power and weight from average representations of a game. By restricting the polyhedron implied by the set of minimal winning and maximal losing coalitions, we can obtain a representation that is also dummy revealing. This might be a hint that they principally could be suited to serve as a measurement for voting power in a certain context. We do not claim that this indeed the case, but propose the challenge to eventually disqualify such a usage rigorously instead. Having the ongoing search for the right index in mind, we want to encourage 
(even) more research with respect to the question of indispensable properties of measurements of power.

The average representations themselves may have other uses too. They conveniently summarize the set of admissible representations of a weighted majority game into a unique representation, which can then be compared to power distributions of the classical power indices. We have already restricted the set of representations in order to obtain a dummy revealing index, but there still may be much more meaningful ways to introduce further restrictions. So our approach might lead to a cornucopia of power indices.

We conclude the paper with a remark on integer weights. A normalization of voting weights is unreasonable if they are to represent the number of shares of a corporation, or the number of members of a political party. In these cases, we require the weights to be integers. However, there is still an interpretation of our indices in these cases, as the following convergence result suggests.

Let us return to the initial example in the introduction, and consider the weighted majority game $v=[2 ; 1,1,1]$. We have said that there are 1176 integer weight vectors being feasible for $v$ with a sum of weights 100 . If we average those

representation we obtain $\left(\frac{100}{3}, \frac{100}{3}, \frac{100}{3}\right)$ or a relative distribution of $\left(\frac{1}{3}, \frac{1}{3}, \frac{1}{3}\right)$, which is no surprise due to the inherent symmetry. Things get a bit more interesting if one considers the weighted majority game $v=[3 ; 2,1,1]$. For a weight sum of 100, we have 1601 different weight vectors, and the averaged relative weight distribution is given by $(0.608832,0.195584,0.195584)$. For a weight sum of 1000 , we obtain 166001 different weight vectors and $(0.610888,0.194556,0.194556)$. For a weight sum of 10000 , we obtain 16660001 different weight vectors and $(0.611089,0.194456,0.194456)$. For a weight sum of 100000 we obtain 1666600001 different weight vectors and (0.6111090.1944460.194446). The averaged relative weight distribution seems to converge to $\left(\frac{11}{18}, \frac{7}{36}, \frac{7}{36}\right)$, which is indeed the average weight index. This can be rigorously proven by numerically approximating the integrals of the definition of the average weight index using grid points only, and considering the limit of finer and finer equally distributed grids. The same is true if an integer valued quota is taken into account. In the limit, we would end up with the average representation index. Also the dummy revealing property can be transfered in this sense.

\section{References}

[Baldoni et al., 2014] Baldoni, V., Berline, N., De Loera, J.A., Dutra, B., Köppe, M., Moreinis, S., Pinto, G., Vergne, M., \& Wu, J. 2014 (January). A User's Guide for LattE integrale v1.7.1. LatteE is available at http://www. math. ucdavic. edu/ latte/.

[Chalkiadakis et al., 2011] Chalkiadakis, G., Elkind, E., \& Wooldridge, M. 2011. Computational aspects of cooperative game theory. Synthesis Lectures on Artificial Intelligence and Machine Learning. Morgan \& Claypool Publishers.

[Colomer \& Martinez, 1995] Colomer, J.M., \& Martinez, F. 1995. The paradox of coalition trading. Journal of Theoretical Politics, 7(1), 41-63. 
[Felsenthal \& Machover, 1998] Felsenthal, D.S, \& Machover, M. 1998. The measurement of voting power: theory and practice, problems and paradoxes. Edward Elgar Cheltenham.

[Freixas \& Kaniovski, 2014] Freixas, J., \& Kaniovski, S. 2014. The minimum sum representation as an index of voting power. European Journal of Operational Research, 233(3), 739-748.

[Holler \& Nurmi, 2013] Holler, M.J., \& Nurmi, H. (eds). 2013. Power, voting, and voting power: 30 years after. Berlin: Springer. xvii, $762 \mathrm{p}$.

[Houy \& Zwicker, 2014] Houy, N., \& Zwicker, W.S. 2014. The geometry of voting power: Weighted voting and hyper-ellipsoids. Games and Economic Behavior, $84,7-16$.

[Kurz et al., 2014] Kurz, S., Napel, S., \& Nohn, A. 2014. The nucleolus of large majority games. Economics Letters, 123(2), 139-143.

[Maschler et al., 2013] Maschler, M., Solan, E., \& Zamir, S. 2013. Game Theory. Cambridge: Cambridge University Press.

[Taylor \& Zwicker, 1999] Taylor, A.D., \& Zwicker, W.S. 1999. Simple games. Desirability relations, trading, pseudoweightings. Princeton, New Jersey: Princeton University Press. 246 p. 